\title{
MINIATURE ANTENNA DESIGN AT LEMA
}

\author{
Anja K. Skrivervik and J.F. Zürcher \\ LEMA, Ecole Polytechnique Fédérale de Lausanne, Station 11, CH-1015 Lausanne, anja.skrivervik@epfl.ch
}

\begin{abstract}
This paper presents the work carried out in the area of antenna miniaturization at the Ecole Polytechnique Fédérale de Lausanne. After a short introduction giving the trends on terminal antennas, design strategies will be presented in the second section. The third section will illustrate these strategies with several practical examples
\end{abstract}

\section{INTRODUCTION}

The need for terminal antennas designed to fulfill the specific need of mobile communications started roughly 25 years ago with the apparition of the $1^{\text {st }}$ generation of mobile phones. Indeed, the mobile phone service had new and stringent requirements for antennas, which differed from the portable radio link systems which were anterior to them, these new requirements being linked to the fact that this new communication service targeted a broad market. Thus, the handheld had to be small enough to be easily carried, of reasonable weight and low cost to manufacture. Initially, the low frequency used gave little degrees of freedom in the antenna design, the only practical solution being a whip where the handheld itself acted as the ground. The strong development of the second generation of mobile phones in the last decade induced an increase of the carrier frequency, giving thus a little more freedom in the antenna design. New mobile services like high speed data transfer (WLAN), Bluetooth, ad hoc networks, mobile peer to peer transfer, as well as the competition for the available frequency spectrum have broadened the range of requirements that are made for the antennas which are used on the mobile terminals. These requirements can be summarized as follows :

- $\quad$ small dimensions

- low weight

- low induced SAR

- low cost

- high efficiency

- capability to handle multiple frequency bands

- broadband

- robust to changes in the environment

- optimize the use of the available channel capacity

The first four requirements are typically "user defined requirements", the others being defined by the service provider or he network.
Of course, depending on the considered service, the relative importance of all these requirements varies a lot. The size is for instance far less critical for WLAN system located in a laptop than for a DCS phone. The bandwidth and capacity however will be far more critical in the former example.

Considering this, the design of terminal antennas is more than ever an art of defining the right compromise between all the requirement for a specific application. In this paper, we will show some design examples for specific situations and propose some solutions to meet the deign requirements listed above.

These examples comprise multifrequency antennas in the second section of this paper, tunable antennas in the third and smart antennas in the fourth. The fifth section is dedicated to the optimization phase of the design process. Some research issues are listed as conclusions in the last section.

\section{DESIGN STRATEGIES}

\subsection{Miniaturization options and their consequences on performance}

Techniques to make antennas smaller have been known for a long time, and many of them are described in standard textbooks (see for instance [1,2] or [3] for more exotic antennas). The principle behind these techniques will be described below, with an emphasis of its effect on the radiation characteristics of the antenna. These techniques have been extensively used in the mobile communication business, where the most interesting results were obtained by combining several of them for the design of one antenna, as is the case of the PIFA and its single and multiband evolutions. References on small antennas for communications can be found in [4,5 and 6$]$.

The simplest and most immediate way to make an antenna smaller than resonant size is loading it with a lumped element, usually a reactance to avoid losses. 
Indeed, the idea is that an antenna smaller than half wavelength will have a strong reactive input impedance that has to be compensated by loading. A classical example is the small loop antenna, which is matched by simply loading it with a capacitor. The effect of this loading will be either to reduce the efficiency of the antenna if the added element has losses, or if the latter are small, to increase the quality factor by enhancing the reactive near fields of the antenna $[7,8]$ and thus reduce its bandwidth. The loading can be performed by adding lumped elements or by adding conductive parts. Like a top hat on a quarter wave monopole.

The loading of the antenna can also be made by modifying the dielectric or magnetic characteristics of the material surrounding it. As the wavelength is shorter in a high permittivity and/or permeability material, the antenna becomes smaller when embedded in such a material. The size reduction will depend on the electric and magnetic characteristics of the material and its shape. Again, assuming that no losses are added, this loading will reduce the bandwidth of the antenna by enhancing its quality factor. This is due to the concentration of the electric field in high permittivity regions (respectively magnetic fields in high permeability regions). Moreover, a higher permittivity or a higher permeability are unfortunately often equivalent to higher dielectric losses.

Another miniaturizing strategy is to make use of ground planes and short circuits. The principle is easily explained by the well-known example of the monopole versus dipole. To be resonant, a dipole must have a length of roughly half a wavelength. This dimension can be halved by replacing one dipole arm by a ground plane, which will in turn create a virtual dipole arm according to image theory.

The principle can be easily extended to planar antennas by adding short circuits to the ground planes, reducing the size of a patch of a quarter of a wavelength. The effect on antenna characteristics is less sever than in the case of loading, as the enhancement of near fields can be kept under control. Losses will however increase due to higher current densities in certain antenna areas.

A more subtle way make an antenna smaller and less bulky is to modify its geometry and shape. A good example is the well-known inverted $\mathrm{L}$ antenna, which was derived from the monopole antenna by just bending it. Adding a short circuit to the inverted L antenna, leads to another popular design, the inverted $\mathrm{F}$ antenna, which is a textbook example of an antenna design combining several miniaturizing techniques.

The effect on the antenna performances of this technique, like the former one, is of two kinds: On one hand it produces greater current concentrations on the antennas, and therefore increases the ohmic losses and decreases the gain of the antennas. On the other hand the techniques used (image effect, position of short circuits, position of slots) can be very frequency sensitive. The antenna bandwidth is thus reduced compared to standard antennas. However, this has not to be the case as some clever designs (IFA and PIFA for instance) show, so the overall effect on antenna performances has to be evaluated from case to case.

Designing an electrically small antenna reduces in most cases to find the best possible compromise between antenna dimensions and radiation characteristics. In most cases today, the specifications are so stringent, that we need to take help of all the antenna's environment (casing, PCB ground planes, etc.) to meet them. In extreme cases, the casing radiates most of the power whereas the actual antenna merely acts as a resonator to set the appropriate working frequency. An example will be shown in section 3 .

\subsection{Multiband antennas}

The venue of new generations of voice services (DCS and UMTS to GSM for instance), and the offer of new services incorporated in phone terminals (like Bluetooth, GPS) require antennas which provides multiband possibilities. Indeed, a multiband antenna solution is often smaller and less costly than a solution with a distinct antenna for each frequency band. Several scenarii can occur, which are all illustrated on the well known PIFA antenna :

- Multi bands but single feed [7-10]. The big advantage of having a common feed point for all the bands is that we do not need to care about mutual coupling problems. The drawback is that the radio front end has to discriminate the signal belonging to different services. This is the usual choice for dual band mobile phone handset antennas. An example of this kind of antenna is shown in figure 1.

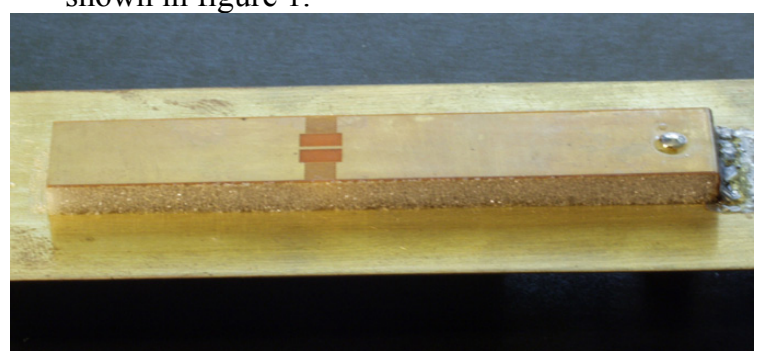

Figure 1 : dual band PIFA antenna (GSM/DCS).

- Multi bands with one feed per frequency band [11]. The drawback is that the mutual coupling between the feed point can be high, which can degrade the overall performances in certain 
circumstances. However, each service is decoupled already at the antenna stage. This scenario is usually chosen when the two offered services are uncorrelated (voice and GSM for instance). This can also be a good choice for frequency bands which are far apart. An example of a double PIFA antenna is shown in figure 2 .

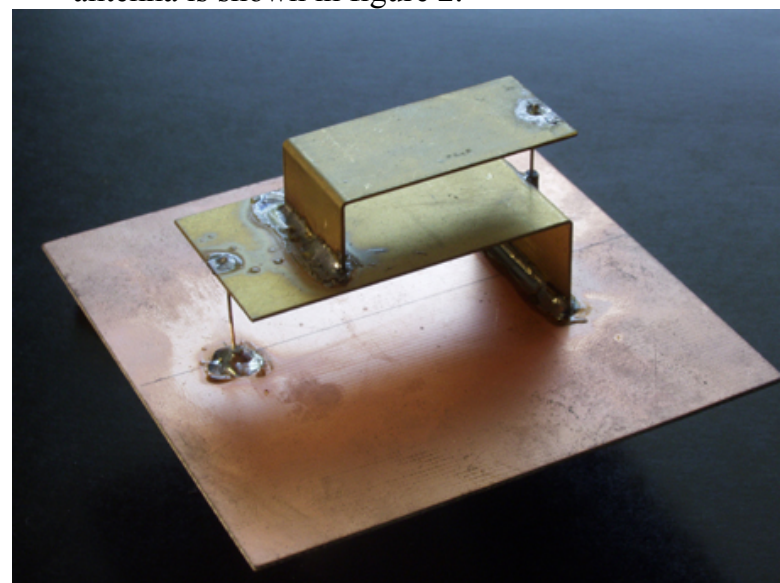

Figure 2 : dual PIFA antenna (GSM/DCS)

- Multi bands with multi-feeds, however less feed than bands [12]. This can be considered as a mixture of two former scenarios, and is often used to cope with the evolution of one type of service. For mobile voice in Europe for instance, one could use a multi band antenna with two feed points, one for the GSM/DCS bands and a second for UMTS. An example covering GSM/DCS on one port and UMTS on the other is shown in figure 3.

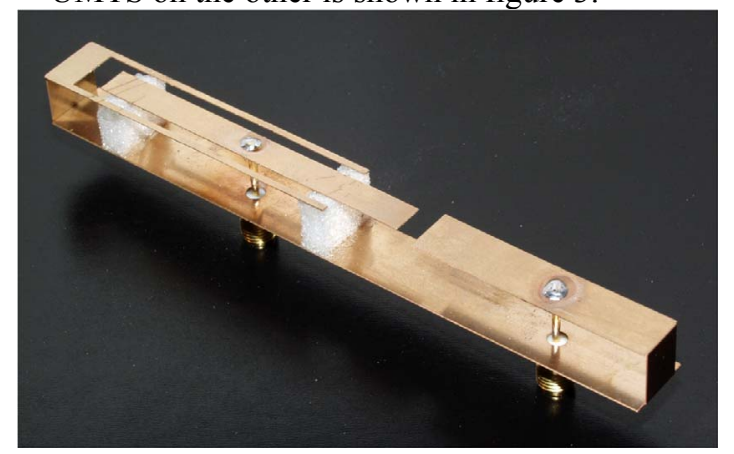

Figure 3 : double PIFA for GSM/DCS (feed point to the left) and UMTS bands

\section{PRACTICAL DESIGN EXAMPLES}

\subsection{Miniature antenna for a wrist watch GPS receiver}

The main challenge in this design was to obtain a circular polarization is the very small space allotted to the antenna in this application. Indeed, the antenna was to be place at the top of the wrist watch, just beneath the hands, and the maximum available volume was of $30 \mathrm{~mm}$ in diameter (meaning a radius of $0.075 \lambda \mathrm{o}$ ) and a height of $1.5 \mathrm{~mm}(0.0075 \lambda \mathrm{o})$. The selected design was circular patch antenna with a slight notch on one axe to obtain circular polarization. In order to reach the specified dimensions, a dielectric substrate with a high permittivity was used ( $\varepsilon r=10.5)$. This value of the dielectric constant was however not enough to obtain a resonance at GPS frequencies within the allotted space, so slots were etched in both axes of the patch in order to further reduce the latter. This option was preferred to selecting a higher permittivity, as the latter would have reduced the achievable bandwidth too much. The Antenna is depicted in figure 4 , and its axial ratio in figure 5 . The impedance bandwidth at $-10 \mathrm{~dB}$ of this antenna is rather small $(0.6 \%)$, as was expected due too the very small size, but it is sufficient if a temperature stabilized dielectric material (like Rogers TMM for instance) is used. The axial ration bandwidth is lightly smaller (Figure 5), and the gain of the antenna was measured at $-5 \mathrm{dBi}$.

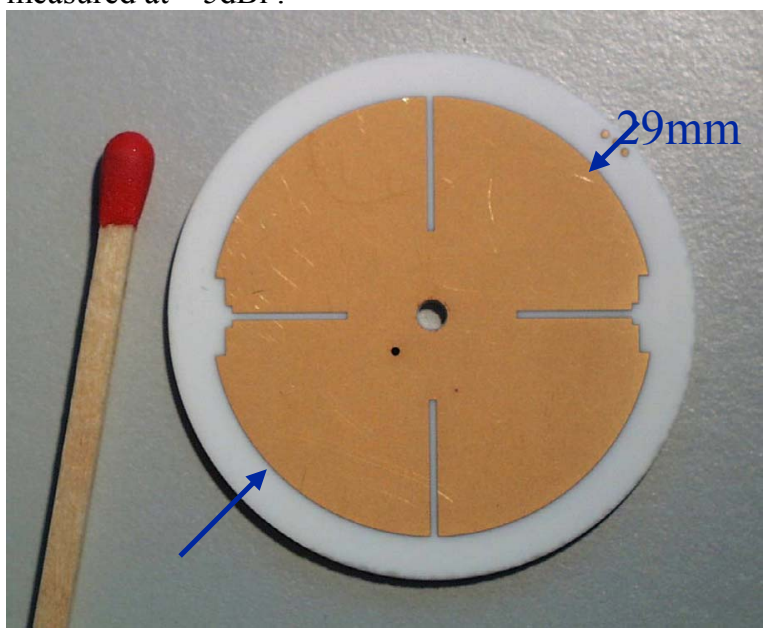

Figure 4 : GPS antenna for a wristwatch

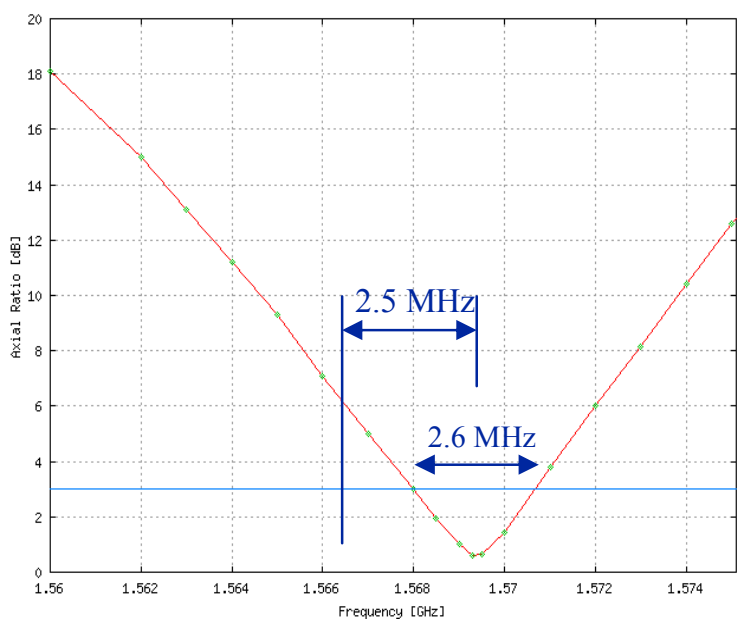

Figure 5 : axial ratio of the GPS antenna of fig. 5 


\subsection{Penta-band antenna for a mobile phone}

In this case, we wanted to design a handset antenna covering five bands (850/900/1800/1900/UMTS) and fitting at the top of a mobile phone handset [13]. The volume allotted to the antenna was $40 \mathrm{~mm} \times 20 \mathrm{~mm}$, with a max height of $8.5 \mathrm{~mm}$, over a ground plane having a max size of $100 \mathrm{~mm} \times 40 \mathrm{~mm}$. As these specifications were quite difficult to match, a specific optimization tool was developed [13] and used to design this antenna. One of the constructed prototypes of the antenna is depicted in figure 6 , the current distribution of the final optimized design in figure 7 and the VSWR of this final antenna in figure 8. In this case, it was not possible to meet all the specifications in the allotted space, and we had to increase very slightly the volume allotted to the antenna.

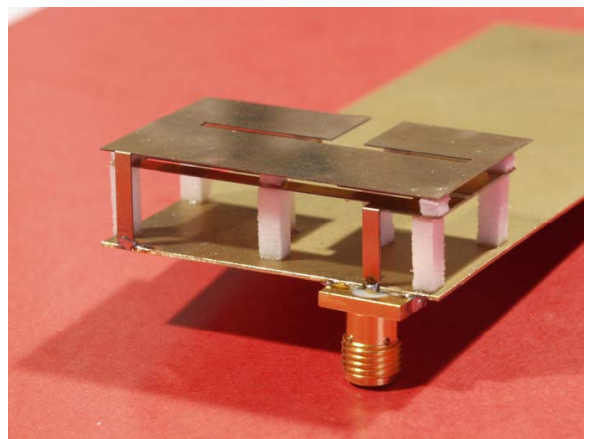

Figure 6: antenna principle

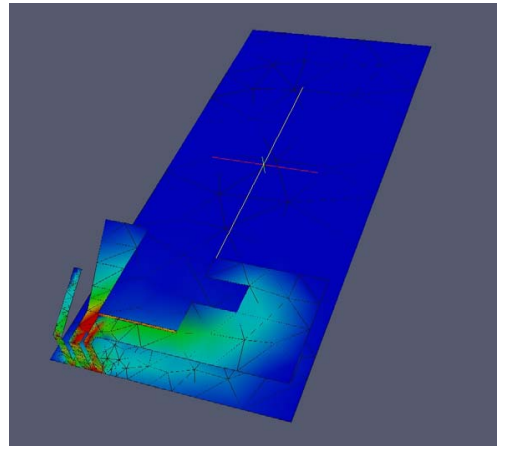

Figure 7: Final optimized structure

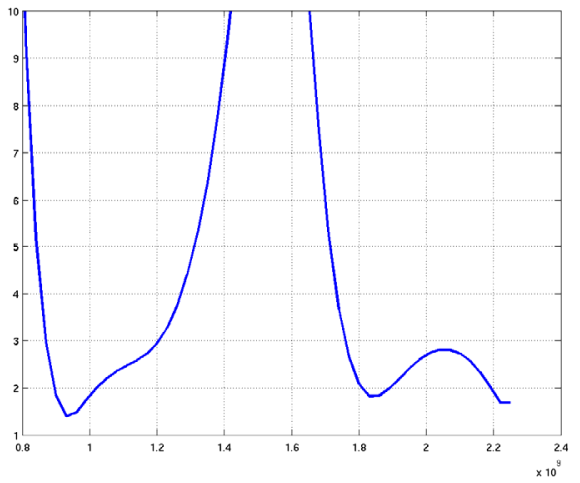

Figure 10: VSWR for the final structure.

\section{CONCLUSIONS}

Some comments on the experience gathered in 15 years of miniature antenna design were presented, illustrated by practical examples.

\section{REFERENCES}

[1] Y.T. Lo and S.W. Lee, Antenna Handbook, Van Nostrand Reinhold, New-York, 1988.

[2] H. Jasik (editor), Antenna engineering handbook, Mc Graw Hill, New-York, 1961.

[3] W.I. Orr, Radio Handbook, Editors and Engineers, Indianapolis, 1975.

[4] K. Fujimoto, A. Henderson, K. Hirasawa, J.R. James, Small Antennas, Research Studies Press, John Wiley and Sons, New-York, 1987.

[5] K. Fujimoto and J.R. James, Mobile Antenna Systems Handbook, Artech House, Norwood, MA, 1994.

[6] A. Kumar, Fixed and Mobile Terminal Antennas, Artech House, Norwood, Ma, 1991.

[7] G.D. Vendelin, Design of Amplifier and Oscillators by the S Parameter Method, Chapitre 2, John Wiley and Sons, New-York 1982.

[8] R.M. Fano, "Thoretical Limitations on the broadband matching of Arbitrary Impedances", Journal of Franklin Institute, Vol. 249, Jan. 1960, pp. 57-83, and Feb. 1960, pp. 1150-1151.

[7] G.Y. Lee and K.L. Wong, "Very low profile bent planar monopole antenna for GSM/DCS dual-band mobile phone", Microwave and Optical Technology Letters, Vol. 34, No. 6, 2001, pp. 406-409.

[8] P.L. Teng, H.T. Chen, "Multi-frequency planar monopole antenna for GSM/DCS/PCS/WLAN operation", Microwave and Optical Technology Letters, Vol. 36, No. 5, 2003, pp. 350-352.

[9] D. Kitchener, M.S. Smith, D.K. Power, J.G. Robson, L.P. Llewellyn, R.H. Johnston, "Multiband terminal and base station antennas for mobile communications", IEE $11^{\text {th }}$ International conference on Antenna and Propagation (ICAP), 17-20. 2001, pp. 68-70.

[10] E. Lee, P.S. Hall and P. Gardner, "Dual band folded monopol/loop antenna for terrestrial communication system, Electronics letters, Vol. 36, 2000, pp. 19901991.

[11] J-F. Zürcher, A. skrivervik, O. Staub, S. Vaccaro, "A compact dual port, dual frequency printed antenna with high decoupling", MOTL, vol. 19, no.2, October 5, 1998, pp. 131-137

[12] F. Nunez, D. Llorens del Rio, J-F. Zürcher, A.K. Skrivervik, "Optimization of a tri-band mobile communication antenna using genetic algorithms", Journées Internationales de Nice sur les antennes (JINA'02), Nice, 12-14 novembre 2002, vol. II, pp. 6568.

[13] F. Nunez, Z. Ying, A. Skrivervik, "Design and optimization of a pentaband terminal antenna", Proc. 18th International Conference of Applied Electromagnetics and Communications, ICECom 2005, Dubrovnik, Croatia, October 12-14, 2005, pp. 33-36. 